\title{
El Arte de hacer y conservar el vino de Francisco Carbonell y Bravo. Un falso original Arte de hacer y conservar el vino, by Francisco Carbonell y Bravo. A false original
}

\author{
MIGUEL IBÁÑEZ RODRÍGUEZ \\ Uva.GIRTraduvino \\ miguel.ibanez@uva.es
}

\begin{abstract}
Resumen: El Arte de hacer y conservar el vino de Francisco Carbonell y Bravo, presentado por su autor como original, es en realidad una traducción. Se trata del primer tratado de enología en español publicado en Barcelona en 1820 por Antonio Brusi. Un hito importante de lo que fue la llegada a España de la ciencia enológica desde Francia. En este trabajo se fija la filiación del texto y el itinerario seguido desde la fuente francesa. Una vez identificado el original francés se hace un análisis comparativo entre la fuente y el tratado de Carbonell, con el fin de identificar qué partes son traducción y cuáles no y cuando lo es hemos valorado en qué medida y cómo se traduce.

Palabras clave: Tratado; vino; Carbonell y Bravo; falso original; fuente.
\end{abstract}

Abstract: Arte de hacer y conservar el vino, by Francisco Carbonell y Bravo, introduced by its author as an original work, is actually a translation. It is the first oenology treatise in Spanish and was published by Antonio Brusi in Barcelona in 1820. It was a landmark in the arrival of the oenological science in Spain from France. This work focuses on the filiation of the text and its journey from its French source. Once identified the French original, an analysis has been carried out, comparing the source text with Carbonell's treatise, in order to identify which parts are a translation and which aren't. Then we have assessed how and to which extent the parts identified as a translation have been translated.

Key words: treatise; wine; Carbonell y Bravo; false original; source.

Fecha de presentación: 08/09/2019 Fecha de aceptación: 19/11/2019.

\section{INTRODUCCIÓN}

Con el presente trabajo vamos a demostrar que el Arte de hacer y conservar el vino de Francisco Carbonell y Bravo, presentado por su autor como original, es en realidad una traducción. Esto que desde la perspectiva actual puede parecer escandaloso, no lo sería tanto en su época en la que los derechos de autor no existían. Más allá de esta circunstancia, nos encontramos ante un texto importante: el primer tratado de enología en español publicado en Barcelona en 1820 por Antonio Brusi. Un hito importante en lo que fue la llegada a España de la ciencia enológica desde Francia.

Pretendemos fijar su filiación y así conocer el itinerario seguido desde la fuente francesa. Para localizar el original partimos de varias posibles fuentes. Con ese fin hemos elaborado un corpus de textos del que damos cuenta al final de este trabajo. A la vista de la genealogía y cronología de los posibles originales y sobre todo como fruto 
del análisis comparativo, hemos identificado el texto de partida utilizado por Francisco Carbonell y Bravo.

Posteriormente hemos hecho un análisis comparativo exhaustivo entre la fuente y el tratado de Carbonell con el fin de identificar qué partes son traducción y cuáles no y cuando lo es hemos valorado en qué medida y cómo traduce.

\section{FRANCISCO CARBONELL Y BRAVO Y SU TRATADO DE 1820}

Nació en Barcelona el 5 de octubre de 1768. Obtuvo muy pronto, a los 17 años, el grado de Doctor en Filosofía por la Universidad de Palma de Mallorca el 4 de septiembre de 1785. Desde el 29 de enero de 1789, con apenas 20 años, dispone del título de Boticario Colegiado. Posteriormente, en 1790, se traslada a Madrid, para dedicarse a la botánica y a la farmacia. Formó parte, como socio colegial del Colegio de Farmacéuticos en febrero de ese mismo año, así como de la Academia de Medicina al año siguiente.

Más adelante se traslada a Huesca, a la Facultad de Medicina de su Universidad, centro donde se graduó en 1795 y con el cual mantuvo siempre una estrecha relación. Continuó sus estudios de medicina en Montpellier donde alcanzó el grado de Doctor en Medicina y Ciencias Naturales el 24 de marzo de 1801. En esta ciudad trabó amistad con Jean Antoine Chaptal, el introductor de la nueva Química en el currículum médico de la citada universidad francesa, y uno de los más importantes promotores de la industria química francesa. Chaptal pretendía aplicar la nueva ciencia a las necesidades regionales del Languedoc, centradas en la viticultura y la industria textil, y Carbonell trató de hacer lo mismo en Cataluña.

A Carbonell se le considera discípulo del químico francés J. L. Proust con quien trabajó durante un año (1802) en el laboratorio que el químico tenía en Madrid. Sus amplios conocimientos dieron como resultado una gran actividad profesional. En 1803 es designado para ocupar la cátedra de Química por la Real Junta de Comercio del Principado de Cataluña, y en 1805 pone en marcha la Escuela Química de Barcelona. Su producción científica se centra en los conocimientos químicos propiamente dichos, de tipo básico, como, por ejemplo, en la aplicación a la medicina de dichos saberes. También desempeñó una labor profesional como revisor de géneros medicinales de la aduana de Barcelona y fue ministro de Protomedicato en la Audiencia de Farmacia.

Sus obras, todas ellas publicadas en Barcelona, son las siguientes: Pharmaciae Elementa Chemiae recientioris fundamentis inixa (1796), Elementos de Farmacia fundados en los principios de química moderna (1801), Química aplicada a las Artes (1816), Curso analitico de química (1818), Ejercicios públicos de química que sostendrán los alumnos de la Escuela Gratuita de esta ciencia establecida en la ciudad de Barcelona (1807 y el Arte de hacer y conservar el vino (1820), considerado como el primer manual de enología científica en español.

Señala en la Advertencia del Arte de hacer y conservar el vino Carbonell (1820: iii) que lo contenido en el mismo corresponde a la recopilación de una serie de artículos de la sección de química de la publicación periódica Memorias de agricultura y artes que se venían editando desde 1815 . Un total de 20 números de dicho periódico, que 
decide reunir en un solo volumen. También la «Noticia sobre la fabricación del vinagre», que se incluye en el tratado, se había publicado en dicha Memoria.

La finalidad del tratado es la poner la química al servicio de la agricultura: «pretendo adelantar la industria de suelo español y ser útil a mis conciudadanos» (Carbonell, 1820: ii), a sabiendas de la importancia que tiene en su «provincia» (Cataluña) y en otras de España la industria del vino.

La obra se presenta como si fuera original. Al comienzo de la misma se anota únicamente a los autores que le «prestan» los materiales, dando a entender que son sus fuentes y no los textos originales desde los que traduce: Chaptal, François de Neufchateau, Olivier de Serres, Guerin, Chevalier y Remer (Carbonell, 1820: 2). Al final, en el Apéndice se anota: «La doctrina que he expuesto en el decurso de este tratado acerca del método de conducir la fermentación, está arreglada a la opinión de los sabios Chaptal y Guerin, y transcrita de sus apreciables obras, que han publicado sobre esta materia (Carbonell, 1820: 279).

En ningún momento de su obra señala Carbonell que se trate de una traducción, tal como era habitual en otros casos, como los recogidos en el apartado Traducciones de nuestra bibliografía, en los que desde la portada se indica explícitamente ese carácter.

\section{UN FALSO ORIGINAL}

Desde un primer momento, L'Art de faire le vin de Chaptal de 1807 se presentaba como principal candidato a ser el posible original del tratado de Carbonell. Sin embargo, para despejar cualquier duda era necesario estudiar la filiación de esta obra francesa y contemplar también como posibles originales otras publicaciones y traducciones con las que está emparentada.

\subsection{El original}

La primera publicación de Chaptal sobre el vino apareció en el diccionario enciclopédico Cours complet d'agriculture del abate Jean François Rozier (1781-1796), este le encargó la entrada o artículo que esta obra dedica al vino. Este trabajo fue retomado por otros autores, entre ellos Cadet de Vaux (1800), y el resultado del mismo fue traducido como veremos al español y también por el mismo Chaptal para escribir L'Art de faire le vin en 1807. Juan Álvarez Guerra tradujo todos los tomos del Cours complet d'agriculture del abate Jean François. El tomo X incluía la entrada vino.

De manera que entre los posibles originales del tratado de Carbonell podría estar: el artículo vino de Chaptal de 1796 del Cours complet d'agriculture, la versión también en francés que de él hizo Cadet de Vaux en 1800 y el tratado que el mismo Chaptal escribió en 1807 a partir del citado artículo: L'Art de faire le vin. También hemos barajado la posibilidad de que Carbonell se hubiera podido servir de la traducción hecha por Manuel Pedro Sánchez Salvador y Berrio del libro de Cadet de Vaux y de la que en el mismo año de 1803 hizo Juan Álvarez Guerra del citado artículo vino al traducir todos los volúmenes del diccionario del Abate Rozier. A continuación haremos un repaso cronológico de estas cinco publicaciones para valorar las posibles vinculaciones con el tratado de Carbonell. 
El Cours complet d'agriculture ou dictionnaire universel d'agriculture de Jean François Rozier que comenzó a publicar en 1781 es una obra que como ya hemos explicado en otros trabajos (Ibáñez, 2018: 31-39) conoció una importante difusión en Francia y en el extranjero. Entre sus subscriptores, además de franceses, aparecen alemanes, italianos, belgas, holandeses y algunos españoles. Y muy pronto fue traducido en su totalidad a otras lenguas europeas, entre ellas al español. En una Reseña (1784) de la obra, cuando aún no se habían publicados todos los tomos, se da noticia de la amplia difusión de la misma. Se presenta como una obra con rigor científico. Está escrita «a pie de obra» y no en el despacho, su autor Jean-François Rozier «a voulu connoître par lui-même les coutumes \& les usages des différents Provinces du Royaume» (Reseña, 1784: 2) y escribe guiado por la «physique, la chymie, \& sur-tout par sa longue habitude en matière de culture» y procura que la teoría quede contrastada por «la pratique la plus solide» (Reseña, 1784: 3). Se trata de un curso de agricultura bajo el formato de diccionario enciclopédico, con ilustraciones y un extenso título: Cours complet d'agriculture théorique, pratique, économique, et de médecine rurale et vétérinaire, suivi d'une méthode pour étudier l'agriculture par principes; ou dictionnaire universal d'agriculture. Es un ejemplo claro del enciclopedismo propio del momento ilustrado en el que surge. La autoría se atribuye a una «Société d'Agriculture» y la redacción al Abbé Rozier («... par une Société d'Agriculture, et rédigé par M. l'Abbé Rozier», se dice en la portada). ¿Cuál fue realmente el papel del Abbé Rozier? Se trata del promotor y editor de la obra, así se presenta desde los primeros tomos publicados, que aparecen con un «Avis de l'éditeur». También es el redactor de los artículos cuya autoría no se indica al final de los mismos, así se señala en una nota del tomo primero: «Les articles de cet ouvrage qui ne sont pas désignés à la fin par des lettres capilales, sont de M. L'Abbé Rozier...»; del resto se indica los autores (M. Mongez, M. Parmentier, M. de Lalause, M. L'Abbé Copineau, M. Falconet, M. Baignière y M. Thorel) y de otros solo las iniciales (M.S., M.N. y M. A.); anotándose en un caso que el autor no quiere aparecer, del que se indican solo las iniciales M.D.M. (Rozier, 1781-1796, I: v).

¿Quién era Jean François Rozier y cómo se gestó esta magna obra sobre agricultura? Se trata de un agrónomo y botánico francés, conocido en Francia como Abbé Rozier y en España como Abate Rozier, que siempre mostró interés por la agronomía, $\mathrm{y}$ en especial por el cultivo de la vid y la elaboración del vino. Conocemos su vida y sus obras gracias a A. J. Dugour (1796: i-xvi) ${ }^{1}$. Nació en Lyon el 24 de enero de 1734 y recibió una esmerada educación en ciencias, bellas artes y teología de mano de los jesuitas y abrazó la vida eclesiástica. Tras la muerte de su padre en 1757 se retiró a las propiedades de la familia que había heredado su hermano mayor y comienza a partir de este momento su interés por la agronomía. Combinando la teoría con la práctica se interesó por los preceptos de los antiguos (Varrón, Columela y Plinio) y los modernos (Olivier de Serres y Duhamel) al tiempo que se interesó con ojo crítico por los métodos practicados en el Mediodía de Francia. Supo relacionar a la agricultura con las ciencias naturales, la física y la química. Fue director la de Escuela de Veterinaria de Lyon,

\footnotetext{
${ }^{1}$ La «Noticia» sobre la vida de la Abbé Rozier de A. J. Dugour, profesor de historia en la Escuela Central, aparece en el volumen X del Cours complet d'agriculture ou dictionnaire universel d'agriculture.
} 
puesto del que al poco tiempo lo apartó su propio mentor Bourgelot por envidias. Perdió su puesto y su pensión y quedó en una situación económica complicada.

Para el presente trabajo lo que más nos interesa es la entrada «vin» del tomo X del Cours complet d'agriculture ou dictionnaire universel d'agriculture, escrita por Jean Antoine Chaptal que es el punto de partida de ese grupo de publicaciones y traducciones, entre las que se encuentra la fuente y el mismo tratado de Carbonell.

En el citado tomo X Jean Antoine Chaptal es presentado como Consejero de Estado y Miembro del Instituto Nacional. Al final de su artículo se le cita de nuevo, y a los dos cargos anteriores ahora se le añade su pertenencia a las Sociedades de Agricultura de los departamentos del Sena, Morbihan, Hérault, etc. Nació el 4 de junio de 1756 en Nojaret (Languedoc) y murió el 30 de julio de 1832 en París, estudió medicina, fue químico y político, nombrado ministro del interior en 1801. Su vida se desarrolló entre Montpellier y París. En su artículo dedicado al vino se sientan las bases de la «teoría de la fermentación» y él mismo es consciente de cómo los nuevos conocimientos están dando lugar a una «lengua científica mediante la cual todos los hombres, y todos los países se comunican entre sí» (Rozier, 1803: 298/211). Por fin hay una lengua común a toda la comunidad vitivinícola, que facilita la transferencia del conocimiento entre todas las regiones que hasta la fecha tenían su vocabulario propio (Ibáñez, 2017: 15-27).

Más tarde Antoine Alexis Cadet-de-Vaux aprovecha este trabajo, el artículo «vin», para realizar a partir de él una versión de la «doctrina» de Chaptal, con el fin de que llegue a los viticultores. Explica que «le citoyen Chaptal», por entonces ministro del interior, «a écrit pour les savants et pour les propriétaires, qui doivent à une éducation libérale d'entendre le langage de la science» y que ahora quiere «qu'on écrive pour le vigneron: mission honorable dont ce ministre me charge» (Cadet de Vaux, 1800: 3).

El éxito del artículo vino de Chaptal en la obra de Rozier, en parte debido a la publicación de Cadet de Vaux, llevó al mismo Chaptal (1807: vii) a publicarlo desgajado en formato de libro y así surgió L'Art de faire le vin en 1807, el que se puede calificar como el primer tratado de enológica. Si hacemos un estudio comparativo podemos ver que este tratado tiene básicamente la misma estructura y los mismos capítulos que la entrada vino de la obra de Rozier. Ahora bien, cuenta con un mayor desarrollo el capítulo IV dedicado a la fermentación del vino, también incluye un capítulo más, el dedicado a la fabricación del vinagre y el resto tiene mayor extensión, mejor método y claridad, según manifiesta el mismo Chaptal (1807: x) en el «Avertissement» que precede la obra. Y así es efectivamente, cuando en los capítulos repetidos hay actualizaciones estas presentan un mayor rigor científico. En este sentido es ya muy significativo que en los añadidos de la introducción de la obra se mencione por primera vez la nueva ciencia del vino: la enología que, llevada de la mano de la química, va a dotar de nombres técnicos a la vinificación y va a facilitar la comunicación (Chaptal, 1807: 12).

Estas publicaciones francesas fueron vertidas al español. Se tradujo el diccionario del Abate Rozier y también la obra de Cadet-de-Vaux. Y como demostraremos más adelante, el tratado de Francisco Carbonell es la traducción de L'Art de faire le vin de Chaptal.

La traducción al español del Cours complet d'agriculture ou dictionnaire universel se comenzó a publicar en España en 1797 y se concluyó en 1803. Su traductor fue Juan 
Álvarez Guerra, que en la portada de la traducción es presentado como «individuo en la clase de agricultura de la Real Sociedad Económica de Madrid» (Rozier, 1797). Juan Álvarez Guerra fue un político y agrónomo español nacido en Zafra (Badajoz) en 1770 y muerto en Madrid en 1845.

La traducción al español resulta ser un diccionario enciclopédico en dieciséis tomos, dedicado a la agricultura. Coincidimos plenamente con Paz Battaner (2001: 225) cuando señala que es un diccionario dentro del «paradigma ilustrado», aunque preferimos incluirlo por tanto dentro de los diccionarios enciclopédicos y no especializados como hace ella. En la Dedicatoria el traductor presenta la obra como «la versión española del libro más completo de Agricultura que se conoce hasta ahora» (Rozier, 1797).

Alcanzó una amplia difusión en su época y años posteriores. El Semanario de agricultura y arte dirigido a los párrocos, que comenzó a publicarse en 1797, lo cita desde sus primeros volúmenes y en ocasiones extracta algunos de sus artículos: «... hemos creído muy necesario extractar este artículo del Diccionario de Rozier...» (1797, II: 21). Braulio Antón señala en su diccionario bibliográfico publicado en 1865 que «el Rozier es harto conocido» y que «contiene millares de artículos, a cual más interesante» y que los periódicos de la época se hicieron eco de él (Antón, 1865: 101-102). Sirvió también de base para un buen número de publicaciones posteriores que «pasan por originales» y para «apreciables manualitos que han salido con el carácter de anónimos» (Antón, 1865: 101).

También en 1803 se publica la traducción de Manuel Pedro Sánchez Salvador y Berrio de L'Art de faire le vin de Cadet-de-Vaux. Su deseo es el de comunicar las nuevas «luces» a todos los cosecheros: «He procurado, que la traducción no desfigure al original, y que su claridad la ponga al alcance de todos los cosecheros» (Cadet de Vaux, 1803: iii-iv).

Hemos comparado con detalle la traducción al español del artículo «vino» con el tratado de Carbonell concluyendo que este último no se sirve de esa traducción. Tampoco se sirve del original francés del citado tratado. Por otro lado, ni la versión original de Cadet-de-Vaux ni la traducción de Manuel Pedro Sánchez Salvador son fuente del texto de Carbonell, tras comparar los textos de manera pormenorizada.

La fuente de la que traduce Carbonell es L'Art de faire de vin de Chaptal. El análisis comparativo que presentamos a continuación lo confirma sin que haya lugar a la menor duda. Además tiene su lógica, pues es de suponer, que Carbonell muy al corriente del tema y sus novedades, prefiere un texto más actualizado como es L'Art de faire de vin de Chaptal que ya habla de la «enología», la nueva ciencia y no el artículo «vin» del Cours complet d'agriculture ou dictionnaire universel d'agriculture. Por otro lado, como ya hemos anotado que Carbonell conoció a Jean Antoine Chaptal en Montpellier mientras estudiaba medicina y ciencias naturales y seguramente pensó en hacer en su provincia catalana lo que el químico francés estaba haciendo en Languedoc: aplicar la química a la agronomía y a la industria textil.

\subsection{Análisis comparativo}

En el Anexo I recogemos un Cuadro comparativo entre la obra de Chaptal de 1807 y la de Carbonell de 1820. Hemos comparado de manera pormenorizada ambas obras capítulo a capítulo y dentro de ellos los artículos o secciones en su caso y a continua- 
ción los textos. La estructura y distribución de ambas obras es muy similar. Hay correspondencia entre los capítulos, artículos y secciones de ambos tratados, salvo en algunos casos puntuales. En cuanto a los capítulos, el tercero de Chaptal pasa a ser una sección (sección II de capítulo IV) en Carbonell, el quinto se convierte en un artículo (artículo V del capítulo V) y el octavo pasa a ser una «Noticia». En el capítulo V de Carbonell, que es traducción del IV de Chaptal, lo que es sección en el original pasa a ser artículo en la traducción (cuatro casos) y al revés, lo que es artículo pasa a sección (ocho casos). En el resto de su tratado, Carbonell respeta las categorías de las subdivisiones de los capítulos del original.

Como resultado del análisis hemos podido comprobar que se producen tres circunstancias diferentes, que en el Cuadro comparativo llamamos así: «Original», «No se traduce» y «Es traducción». De los pasajes escritos por Carbonell, decimos que es «Original». Cuando indicamos que «No se traduce» se trata de partes de la obra de Chaptal que Carbonell decide no traducir. Del resto, es decir de lo vertido al castellano por Carbonell tomando como texto de partida el de Chaptal, decimos que «Es traducción», ya veremos más adelante en qué grado y medida.

Según se puede ver en el Cuadro comparativo, es «Original» en el tratado de Carbonell: la Advertencia (2 páginas ${ }^{2}$ ), el «Prólogo» ${ }^{3}$ (pp. 1-2), el Capítulo primero (pp. 935), el Capítulo III (pp. 58-88), el apartado «Máquinas para estrujar las uvas» del Capítulo IV (pp. 110-115), un breve fragmento del Capítulo V (pp. 147-148), el Capítulo VIII (pp. 224-231), el Capítulo IX (pp. 231-251), la Sección IV de la «Noticia ..» (pp. 274-278) y el «Apéndice al Arte de hacer y conservar el vino» (pp. 279-289). Son un total de 111 páginas, lo que representa el 36,39\% del tratado, que tiene un total de 305 páginas (289+16 del apéndice). Por lo tanto, el resto, el 63,6\% de la obra de Carbonell «Es traducción» del tratado de Chaptal.

Del total de las 367 páginas que comprenden el tratado de Chaptal, se quedan sin traducir 74 («No se traduce»), distribuidas de la siguiente manera: «Advertencia» (pp. viixiv), introducción del Capítulo IV (pp. 96-99), Sección IV del Capítulo VI «Des vasseaux propres à conserver les vins» (pp. 241-248), el Capítulo IX «Des vertus du vin» (pp. 311-318) y Capítulo X «Des principes contenus dans le vin et de sa distillation» (pp. 318-367). Las páginas no traducidas representan el 20,16\% del tratado de Chaptal. Si descontamos las páginas de la «Advertencia», en la que el químico francés explica la génesis de su obra y que por tanto no procedería su traducción, sería el $18 \%$ lo no traducido. Por lo tanto, Carbonell traduce el $82 \%$ de la obra de Chaptal. En términos actuales diríamos que la «fusila», aunque tal calificativo no sería aplicable para aquellos tiempos.

No descartamos que parte o todo de lo que hemos considerado original no sea también traducción de otras fuentes francesas distintas del tratado de Chaptal. A fecha de hoy lo desconocemos. Carbonell comienza explicando el interés del libro y su contenido, en lo que hemos llamado «prólogo». El capítulo primero titulado «Ideas y reflexiones generales sobre la viña» (pp. 9-35) consta de varias secciones: sección primera «Consideraciones generales» en la que se habla de la necesidad de «un tratado comple-

\footnotetext{
${ }^{2}$ No está paginada

${ }^{3}$ El texto aparece sin título alguno, nosotros lo denominamos prólogo.
} 
to de la historia natural de esta planta» y cita a autores como Bonnet, Duhamel Columela, Olivier de Serres, Roger Schabol o Arthur Joung; S. II «Del producto de la viña» en la que hace referencia a los romanos, Columela y al Abate Rozier entre otros; S. III «Observaciones botánico-meteorológicas sobre la viña», un tanto dispersa, se ocupa, entre otras cosas, de las heladas en la viña; $\mathrm{S}$. IV «De los medios de renovar la viña»; $\mathrm{S}$. V «De la viña abandonada a sí misma, y de algunos experimentos que faltan hacer sobre este punto» y S. VI «De la viña como adorno de las casas, de los jardines, etc.».

El otro capítulo de cosecha propia es el tercero que se titula «De la construcción y conservación de las bodegas, de los lagares, y de los toneles» (pp. 58-88). Esta temática, en particular lo relativo a la bodega y lagares, no está en el tratado de Chaptal. Sí que incluye un apartado sobre recipientes para el vino, en la Sección IV del Capítulo VI titulada «Des vaisseaux propres à coserver les vins» (p. 241) que no es traducido por Carbonell pues sus contenidos ya los incluye en el capítulo tercero. Este incluye cuatro secciones: S. I «Descripción de un establecimiento para la cosecha de vin»; S. II «De las bodegas»; S. III «De los lagares»; S. IV «De los toneles».

Dentro del capítulo IV es original el apartado dedicado a las máquinas para estrujar las uvas (pp. 110-118). Se trataría de máquinas nuevas y Carbonell querría darlas a conocer. En la sección IV del capítulo V Carbonell introduce un pequeño fragmento original (pp. 147-148) sobre el principio colorante.

También son originales el capítulo VIII (pp. 224-231) que se ocupa de los vinos artificiales y el capítulo IX (pp. 231-251) dedicado a la policía judicial química del vino. En el primero se explica cómo hacer vino con otros frutos y productos diferentes a la uva, así se puede hacer vinos artificiales de guindas, grosellas, moras silvestres, naranjas, del zumo de caña de azúcar, etc. En el segundo se explica cómo se persigue la falsificación de vinos, pues hay traficantes que por diferentes medios les aplican color, olor y sabor de manera artificial.

Y por último también son originales la sección IV titulada Policía judiciaria química del vinagre (pp. 274-278), del que se explican diferentes formas de falsificarla, y el Apéndice al arte de hacer y conservar el vino (pp. 279-280).

Como ya hemos anotado, son 74 las páginas de Chaptal que Carbonell deja sin traducir. No traduce el «Advertissement» (pp. vii-xiv) por razones evidentes, que ya hemos indicado antes, y la sección IV titulada «Des vasseaux propres à conserver les vins» (pp. 241-248), por ser contenidos ya recogidos en el capítulo III. Tampoco traduce la introducción del capítulo IV (pp. 96-99), sin que conozcamos el motivo. La parte más sustanciosa que queda sin traducir es el capítulo IX (pp. 311-318) que se ocupa «Des vertus du vin» y el más extenso capítulo X (pp. 318-367) titulado «Des principes contenus dans le vin et de sa distillation». Aparentemente no hay razones para que no se tradujeran estos dos capítulos, sin duda, de interés. Tal vez porque le resultan demasiado teóricos a Carbonell que escribe para el viticultor y no para el químico o enólogo. En el capítulo IX se dice que el vino es una bebida tónica, fortificante, nutritiva y saludable (Chaptal, 1807: 311) y el $X$ explica los diferentes componentes del vino: acidez, alcohol, tartárico, aroma o buqué y principio colorante. Al hablar del alcohol explica también la destilación del vino, en particular las mejoras que se han producido para obtención del alcohol del vino. 


\subsection{La traducción}

Como ya hemos anotado más arriba, el 63,6\% de la obra de Carbonell está traducida del tratado de Chaptal. Traduce les «Vues générales», que viene a ser una introducción y todos los capítulos seguidos (I, II, III, IV, V, VI, VII, VIII), salvo el IX y el X. Cuando hablamos de traducción lo decimos en el sentido de lo que se entiende por traducción, tal como se puede comprobar en los tres fragmentos del Anexo II, en los que incluimos el texto de partida y el de llegada.

Cuando traduce lo hace muy pegado al original y con pequeñas variaciones para acomodar el texto al público español. Por ejemplo, cuando Chaptal se refiere a regiones concretas de Francia, aunque no siempre, Carbonell las omite sin más: «On a observé, en Champagne, que le raisin cueilli le matin...» (Chaptal, 1807: 103) / «En varias partes se ha observado, que las uvas cogidas por la mañana...» (Carbonell, 1820: 120). Sustituye «En Bourgogne, en Champagne et dans le Bordelais, ...» (Chaptal, 1807: 14) por «En varios parajes...» (Carbonell, 1820: 96) en la traducción. Algo similar hace en este otro caso: «En Champagne, dans les cantons où l'on récolte des vins rouges..» (Chaptal, 1807: 221) / «En algunos parajes en que se fabrican vinos colorados, hacia...» (Carbonell, 1820: 195). Introduce referencias a España buscando la localización cuando en el original solo se cita Francia: «De tous les pays, celui, sans doute, qui présente la situation la plus heureuse, c'est la France...» (Chaptal, 1807: 17) / «Entre todos los países verdaderamente la España y la Francia son los que presentan la mejor situación...» (Carbonell, 1820: 38). De este modo deslocaliza el texto para que sirva también para el caso español. En alguna ocasión introduce algún ejemplo de su propia región, para buscar cierta adaptación que no llega a conseguir ante la gran cantidad de referencias geográficas francesas del original que traslada a su obra, como la siguiente: «Así es que las uvas de las orillas del Cher y del Loire en la Turena son muy negras...» (Carbonell, 1820: 146). Otras veces se explicita que es en Francia, para no confundir al lector español: «En 1769, les raisins encore verts, dit Rozier, ont été surpris para les gélées des...» (Chaptal, 1807: 62) / «En Francia en el año 1769 estando las uvas verdes todavía, dice Rozier, sobrevinieron unas...» (Carbonell, 1820: 92).

En la parte traducida hay varias notas a pie de página que son de Carbonell, que no están en el original y añaden más información sobre la temática tratada y hacen remisión a otras partes del tratado. Al final del artículo IV del capítulo V Carbonell (1820: 167) introduce una extensa cita para presentar y destacar el interés para los «labradores y cosecheros instruidos» de una serie de tablas con experiencias que traslada directamente de Chaptal. En el artículo V de ese mismo capítulo «transcribe», en realidad traduce (Carbonell, 1820: 182-185), en otra nota a pie de página dos artículos, uno dedicado al enómetro y el otro al gleucómetro, el primero de Bosch y el segundo de Permantier, ambos del Curso completo de agricultura publicado en Francia en 1809. En el citado artículo $\mathrm{V}$ aún añade dos citas más en las que incluye información sobre la fabricación del aguardiente (Carbonell, 1820: 191-192). Y en la última cita (Carbonell, 1820: 1999) hace remisión a la sección IV del capítulo III en lo relativo a azufrar los toneles. 
Traduce alguna de las citas latinas manteniendo el texto latino (Carbonell, 1820: 3), tal vez porque los destinatarios de su obra son menos doctos que los del original. Escribe para labradores y cosecheros instruidos.

Observamos que en la traducción hay tendencia a evitar los tecnicismos recurriendo a la perífrasis. Un ejemplo de ello lo vemos en el fragmento 3 del Anexo II que incluimos al final. En el título de la sección del original francés se dice «De la maladie du vin appelée graise» (Chaptal, 1807: 254) y Carbonell (1820: 211) traduce «de la enfermedad o alteración que sufre el vino cuando se espesa como el aceite». En otro caso en lugar de incluir acescencia en la traducción, Carbonell (1820: 215) prefiere decir: «De la alteración o enfermedad de agriarse el vino espontáneamente» para traducir «De la acescense spontanée du vin» (Carbonell, 1807: 259). Es reacio pues Carbonell a introducir neologismos. Evita el neologismo enólogo, que ya se había introducido en 1803 en la traducción de Manuel Pedro Sánchez Salvador y Berrio y en la de Juan Álvarez Guerra (Ibáñez, 2017): «Les écrits des oenologues fourmillent de récettes...» (Chaptal, 1807: 268) / «Finalmente en los escritos de los varios autores que han tratado del vino se encuentran...» (Carbonell, 1820: 221). Lo mismo hace con «bouquet» (Chaptal, 1807: 121) que traduce Carbonell (1820: 120 y 160) como «principio oloroso» o «fragancia» y con «chapeau» (Chaptal, 1807: 199) que vierte como «costra o capa» (Carbonell, 1820: 179).

Tal vez hay un deseo de no delatar que traduce, no quiere extranjerizar no vaya a ser que se sospeche que es traducción. Y con la misma intención posiblemente utiliza voces castizas como «majuelo» (Carbonell, 1820: 39) o espíritu que alterna con alcohol: «... deben producir unos vinos muy espirituosos, supuesto que el azúcar es necesario a la formación del alcohol o espíritu del vino...» (Carbonell, 1820: 39).

Nos parecía importante valorar qué hace Carbonell cuando en el texto de partida Chaptal habla en primera persona. Pues recurre a una tercera persona o la anula, llegando incluso a suplantarlo descaradamente. Cuando Chaptal (1807: 304) dice «Une expérience que j'ai faite en 1781, et qui se trouve dans les Mémoires de l'académie...», Carbonel (1820: 304) traduce: «Un experimento que practicó Chaptal en el año 1781, según consta en las memorias...», sustituyendo el yo por un Chaptal en tercera persona. Otros ejemplos similares: «J'ai fait quelques expériences» (Chaptal, 1807: 120) / «Chaptal hizo varios experimentos...» (Carbonell, 1820: 120); «J'ai exposé des vins vieux...» (Chaptal, 1807: 263) / «Chaptal puso vino rancio...» (Carbonell, 1820: 217) y «Je dirai» (Chaptal, 1807: 134) / «... vamos a tratar...» (Carbonell, 1820: 136). La explicación en nota a pie de página del término alcohol lo comienza Chaptal (1807: 141) en primera persona: «J'emploie ici le mot alcohol, quoique...» y Carbonell (1820: 140) también pero del plural: «Empleamos el nombre de alcohol, aunque ...».

Chaptal (1807: 289) usa la primera persona y dice haber visto el procedimiento que explica la fabricación del vinagre de la cerveza. Carbonell (1820: 262), por su parte, pasa a la tercera y suprime ese detalle: «Je décrirai le procédé que j'ai vu exécuter dans le Nord de la France (ci-devant Belgique)»/«El método que se practica en la Bélgica para la fabricación del vinagre de cerveza...».

En otro momento de la obra Chaptal (1807: 181) anota: «La théorie que je viens d'établir sur la fermentation spiritueuse...» y Carbonell (1820: 164) traduce, haciéndose, 
de manera sorprendente, partícipe de la creación de la teoría de la fermentación: «La teoría que acabamos de establecer acerca la fermentación espirituosa...». Y en otros casos suplanta sin reparos al mismo Chaptal (1807: 51): «Je crois qu'il faut faire une grande différence...» y Carbonell (1820: 56) dice: «Creo que es necesario hacer...».

Contrariamente a lo que hace Carbonell, lo habitual en su tiempo era indicar que se trataba de una traducción. Hemos anotados varios casos de fechas anteriores y próximas al tratado de Carbonell en el apartado «Traducciones», que hemos recogido después de las referencias bibliográficas, al final de este trabajo. Son de agronomía, pero en esa época eran muy abundantes las traducciones al español de textos fundamentalmente del francés de contenido científico y técnicos (Ibáñez, 2015).

Una de las traducciones anotadas es la que realiza Juan Álvarez Guerra del Curso completo o diccionario universal de agricultura teórica, práctica, económica, y de medicina rural y veterinaria del Abate Rozier, realizada entre 1797 y 1803. El tomo dieciséis contiene la traducción de Chaptal, del artículo vino a partir del cual, como hemos anotado, escribe el citado autor L'Art de faire le vin. De manera que, teniendo conocimiento de ello seguramente, Carbonell lo traduce, a pesar de que ya circulaba una traducción de su texto en España.

\section{CONCLUSIONES}

El Arte de hacer y conservar el vino de Carbonell es un falso original. El 63,6\% es traducción de L'Art de faire le vin de Chaptal, tratado de cuyos contenidos traduce un $82 \%$. No recrea, ni reescribe el original, lo traduce en el sentido exacto del término, tratando de trasladar sus contenidos de manera fiel al español. La estructura y distribución entre el original y la traducción es muy similar. Hay correspondencia entre los capítulos, artículos y secciones de ambos tratados, salvo en algunos casos puntuales.

Lo hace de manera deliberada, busca estrategias que no delaten que traduce, así suplanta al autor cuando habla en primera persona o la evita recurriendo a la tercera persona o anulándola.

Domestica el texto para reforzar su autoría, suprimiendo referencias geográficas francesas. Deslocaliza el texto para que sirva también para el caso español y en alguna ocasión introduce algún ejemplo de su propia región. Introduce, de cosecha propia, varias notas para ampliar el texto o aclarar remisiones.

Evita los tecnicismos recurriendo a las perífrasis, pues suponían la transferencia de extranjerismos, galicismos que lo podían delatar. Además por su formación y conocimiento de la materia sabía sin duda que ya circulaban en España una traducción de un estadio anterior del original francés del que se sirve y que era habitual que cuando se trataba de una traducción se indicaba en la portada de la obra.

Más allá de esta circunstancia, el texto es importante, ya que se trata del primer manual de enología en español, de indudable interés para los labradores y cosecheros para los que destina su obra. Pensando en ellos traduce algunas citas latinas, cosa que no se hace en el original. 


\section{REFERENCIAS BIBLIOGRÁFICAS}

ANTÓN RAMÍREZ, Braulio (1865): Diccionario de bibliografia agronómica y de toda clase de escritos relacionados con la agricultura, seguido de un índice de autores y traductores con algunos apuntes biográficos, Madrid, Impr. y Est. de M. Rivadeneyra.

BAtTANeR, Paz (2001): "La traducción de los diccionarios de especialidad: Estudio de algunos casos del siglo XIX», en Jenny Brumme, ed., La historia de los lenguajes iberorrománicos de especialidad. La divulgación de la ciencia, Barcelona, IULA, Universidad Pompeu Fabra, pp. 224-241.

CADET DE VAuX, Antoine-Alexis (1800): L'Art de faire le vin, d'après la doctrine de Chaptal: instruction destinée aux vignerons, París, Bureau de la Décade philosophique.

CAdet De VAuX, Antoine Alexis (1803): Arte de hacer el vino, traducido por Manuel Pedro Sánchez Salvador y Berrio, Pamplona, Viuda de Longás.

Chaptal, Jean-Antoine (1807): L'Art de faire le vin, París, Deterville.

Dugour, A. J. (1796): «Notice sur la vie et les écrits de l'Abbé Rozier», en François Rozier, Cours complet d'agriculture théorique, pratique, économique et de médecine rurale et vétérinaire... ou dictionnaire universel d'agriculture, París, Delalain fils, 10, pp. i-xvi.

IBÁÑEZ RoDRíGUEZ, Miguel (2015): «La traducción científico-técnica francés-español en el ámbito de la enología (1750-1850)», Çedille: Revista de Estudios Franceses, 11, pp. 273-311.

IBÁÑEZ RODRÍGUEZ, Miguel (2017): «L'Art de faire le vin y su traducción al español: gestación de un primer vocabulario técnico del vino (1786-1845)», Hikma, 16, pp. 9-33.

IBÁÑEZ RoDRíGUEZ, Miguel (2018): El tratado de la vid de Louis Dussieux y el tratado del vino de Jean Antoine Chaptal de 1796 conservados en la Biblioteca de San Millán: contextualización y estudio de la traducción de 1803, San Millán de la Cogolla, Cilengua, Fundación San Millán.

RESEÑA (1784) de Cours complet d'agriculture théorique, pratique, économique, et de médcine rurale et vétérinaire; suivi d'une méthode pour étudier l'agriculture par principes ou dictionnaire universel d'agriculture, par une Société d'agriculteurs, et rédigé par M. l'Abbé Rozier, París, De l'imprimerie de Cl. Simon.

RozIER, Jean François (1781-1796): Cours complet d'agriculture, théorique, pratique, économique, et de médecine rurale et vétérinaire suivi d'une méthode pour étudier l'agriculture par principes ou dictionnaire universel d'agriculture, París, Rue et Hôtel Serpente, tomos I-X.

RozIER, Abate (1797-1803): Curso completo o diccionario universal de agricultura teórica, práctica, económica, y de medicina rural y veterinaria. Escrito en francés por una Sociedad de Agrónomos, y ordenado por el Abate Rozier. Traducido al castellano por Don Juan Álvarez Guerra, individuo de la clase de agricultura de la Real Sociedad Económica de Madrid, Madrid, Imprenta Real, 16 tomos [el tomo XVI incluye los artículos «vid/viña» $\mathrm{y}$ «vino»].

Semanario de agricultura y artes dirigido a los párrocos (1797): Madrid, Imprenta de Villalpando, tomos I y II.

\section{TRADUCCIONES}

Abu Zacaria Ihaia Aben Mohamed Been Ahmed Ebn Ahmed Ebn El Awam (1802): Libro de agricultura, traducido al castellano y anotado por D. Josef Antonio Ban- 
queri, prior claustral de la catedral de Tortosa, individuo de la Real Biblioteca de S. M. y académico de número de la Real Academia de la Historia, Madrid, Imprenta Real, dos tomos.

Agustín, Miguel (1749): Libro de los secretos de agricultura, casa de campo y pastoril traducido de lengua catalana en castellano por Fr. Miguel Agustín del libro que él mismo compuso, Barcelona, Casa de Pedro Escuder.

CAdeT DE VAuX, Antoine Alexis (1803): Arte de hacer el vino, traducido por Manuel Pedro Sánchez Salvador y Berrio, Pamplona, Viuda de Longás.

Columela, Lucio Junio Moderato (1824): Los doce libros de agricultura, que escribió en latín Lucio Junio Moderato Columela, traducidos al castellano por D. Juan María Álvarez de Sotomayor y Rubio, Madrid, Miguel de Burgos.

ROZIER, Abate (1797-1803): Curso completo o diccionario universal de agricultura teórica, práctica, económica, y de medicina rural y veterinaria. Escrito en francés por una Sociedad de Agrónomos, y ordenado por el Abate Rozier. Traducido al castellano por Don Juan Álvarez Guerra. Individuo de la clase de agricultura de la Real Sociedad Económica de Madrid, Madrid, Imprenta Real, 16 tomos.

\section{CORPUS POR ORDEN CRONOLOGICO}

Chaptal, Jean Antoine (1796): «Vin», en Cours complet d'agriculture théorique, pratique, économique et de médecine rurale et vétérinaire ou dictionnaire universel d'agriculture, París, Librairie d'Education et des Sciences et Arts, X, pp. 284-377.

CADET DE VAuX, Antoine-Alexis (1800): L'Art de faire le vin, d'après la doctrine de Chaptal: instruction destinée aux vignerons, París, Bureau de la Décade philosophique.

CAdET DE VAuX, Antoine Alexis (1803): Arte de hacer el vino, traducido por Manuel Pedro Sánchez Salvador y Berrio, Pamplona, Viuda de Longás.

CHAPTAL, Jean Antoine (1803): «Vino», en Curso completo o diccionario universal de agricultura teórica, práctica, económica y de medicina rural y veterinaria, escrito en francés por una sociedad de agrónomos y ordenada por el Abate Rozier, traducido al castellano por Don Juan Álvarez Guerra, individuo de mérito en la clase de agricultura de la Real Sociedad Económica de Madrid, Madrid, Imprenta Real, XVI, pp. 292-386.

Chaptal, Jean-Antoine (1807): L'Art de faire le vin, París, Deterville.

CARBONELl y BRAVO, Francisco (1820): Arte de hacer y conservar el vino, con una noticia acerca la fabricación del vinagre, Barcelona, Antonio Brusi [contiene una Adición al apéndice del Arte de hacer y conservar el vino de F. Carbonell y Bravo, Barcelona, Antonio Brusi, 1824].

ANEXO I: CUADRO COMPARATIVO

\begin{tabular}{|l|l|l|}
\hline Chaptal 1807 & Carbonell 1820 & ¿Es traducción? \\
\hline Avertissement (pp. vii-xiv) & & $\begin{array}{l}\text { No se traduce. } \\
\text { Chaptal explica la génesis de } \\
\text { su obra. }\end{array}$ \\
\hline & Advertencia (2 pp.). & $\begin{array}{l}\text { Original. } \\
\text { Carbonell explica la génesis } \\
\text { de su obra. }\end{array}$ \\
\hline & «Prólogo» (pp. 1-2). & $\begin{array}{l}\text { Original. } \\
\text { Se explica el interés del libro } \\
\text { y su contenido. }\end{array}$ \\
\hline
\end{tabular}




\begin{tabular}{|c|c|c|}
\hline Vues générales (pp. 1-13). & Introducción (pp. 2-9). & $\begin{array}{l}\text { Es traducción. Traduce la } \\
\text { cita en latín (p. 3). Hay un } \\
\text { fragmento que no traduce } \\
(\mathrm{pp} .8-12)\end{array}$ \\
\hline & $\begin{array}{l}\text { Capítulo Primero. } \\
\text { Ideas y reflexiones generales sobre } \\
\text { la viña (pp. 9-35). }\end{array}$ & Original $^{4}$. \\
\hline $\begin{array}{l}\text { Chapitre Premier } \\
\text { Du raisin, considéré dans ses } \\
\text { rapports avec le sol, le climat, } \\
\text { l'exposition, les saisons, la cul- } \\
\text { ture, etc. (pp. 13-55). }\end{array}$ & $\begin{array}{l}\text { Capítulo II } \\
\text { De la uva considerada con relación } \\
\text { al terreno, al clima, a la exposición, } \\
\text { a las estaciones y al cultivo (pp. 35- } \\
\text { 58). }\end{array}$ & $\begin{array}{l}\text { Es traducción. } \\
\text { Coincidencia plena. }\end{array}$ \\
\hline $\begin{array}{l}\text { Section Première } \\
\text { De l'influence du climat sur le } \\
\text { raisin (pp. 15-21). }\end{array}$ & $\begin{array}{l}\text { S. I } \\
\text { Del influjo del clima sobre las uvas } \\
\text { (pp. 36-40). }\end{array}$ & $\begin{array}{l}\text { Es traducción. } \\
\text { Coincidencia plena. }\end{array}$ \\
\hline $\begin{array}{l}\text { Section II } \\
\text { De l'influence du sol sur le raisin } \\
\text { (pp. 21-30). }\end{array}$ & $\begin{array}{l}\text { S. II } \\
\text { Del influjo del terreno sobre las } \\
\text { uvas (40-44). }\end{array}$ & $\begin{array}{l}\text { Es traducción. } \\
\text { Coincidencia plena. } \\
\text { No se traducen las citas } \\
\text { latinas (Chaptal p. } \\
\text { 12/Carbonell p. 40). }\end{array}$ \\
\hline $\begin{array}{l}\text { Section III } \\
\text { De l'influence de l'exposition de } \\
\text { la vigne sur le raisin (pp. 30-41). }\end{array}$ & $\begin{array}{l}\text { S. III } \\
\text { Del influjo de la exposición de la } \\
\text { viña sobre las uvas (pp. 45-51). }\end{array}$ & $\begin{array}{l}\text { Es traducción. } \\
\text { Coincidencia plena. }\end{array}$ \\
\hline $\begin{array}{l}\text { Section IV } \\
\text { De l'influence des saisons sur le } \\
\text { raisin (pp. 41-47). }\end{array}$ & $\begin{array}{l}\text { S. IV } \\
\text { Del influjo de las estaciones sobre } \\
\text { las uvas (pp. 51-54). }\end{array}$ & $\begin{array}{l}\text { Es traducción. } \\
\text { Coincidencia plena. }\end{array}$ \\
\hline \multirow[t]{2}{*}{$\begin{array}{l}\text { Section } \mathbf{V} \\
\text { De l'influence de la culture sur le } \\
\text { raisin (pp. 47-55). }\end{array}$} & $\begin{array}{l}\text { S. V } \\
\text { Del influjo del cultivo sobre las } \\
\text { uvas (pp. 54-58). }\end{array}$ & $\begin{array}{l}\text { Es traducción. } \\
\text { Coincidencia plena. }\end{array}$ \\
\hline & $\begin{array}{l}\text { Capítulo III } \\
\text { De la construcción y conservación } \\
\text { de las bodegas, de los lagares, y de } \\
\text { los toneles (pp. 58-88). }\end{array}$ & Original. \\
\hline $\begin{array}{l}\text { Chapitre II } \\
\text { Du moment le plus favorable } \\
\text { pour la vendange, et des moyens } \\
\text { d'y procéder (pp. 56-73). } \\
\end{array}$ & $\begin{array}{l}\text { Capítulo IV } \\
\text { De la vendimia (pp. 89-109). }\end{array}$ & $\begin{array}{l}\text { Es traducción. } \\
\text { Coincidencia plena. }\end{array}$ \\
\hline $\begin{array}{l}\text { Du moment le plus favorable } \\
\text { pour la vendange, et des moyens } \\
\text { d'y procéder (pp. 56-73). }\end{array}$ & $\begin{array}{l}\text { S. I } \\
\text { Del momento más favorable para la } \\
\text { vendimia, y de los medios de prac- } \\
\text { ticarla (pp. 89-98). }\end{array}$ & $\begin{array}{l}\text { Es traducción. } \\
\text { Coincidencia plena. }\end{array}$ \\
\hline $\begin{array}{l}\text { Chapitre III } \\
\text { Des moyens de disposer le suc du } \\
\text { raisin à la fermentation (73-95). }\end{array}$ & $\begin{array}{l}\text { S. II } \\
\text { De los medios de disponer el zumo } \\
\text { de las uvas para la fermentación } \\
(98-109)\end{array}$ & $\begin{array}{l}\text { Es traducción. } \\
\text { Coincidencia plena. } \\
\text { Carbonell trasforma en una } \\
\text { sección un capítulo del } \\
\text { original. } \\
\text { Hace una traducción libre de } \\
\text { la nota a pie de página (Chap- } \\
\text { tal p. 73/Carbonell p. 99). }\end{array}$ \\
\hline
\end{tabular}

\footnotetext{
${ }^{4}$ De todas formas, no descartamos que lo tome de alguna fuente francesa que no hemos podido identificar.
} 


\begin{tabular}{|c|c|c|}
\hline & $\begin{array}{l}\text { Máquinas para estrujar las uvas (pp. } \\
110-118 \text { ) } \\
\text { Máquina de Lavoipierre (pp. 110- } \\
115 \text { ) } \\
\text { Máquina Mauro Amatller (pp. } \\
\text { 115-118) }\end{array}$ & $\begin{array}{l}\text { Original. } \\
\text { Sin duda, son máquinas nuevas } \\
\text { y quiere darlas a conocer. }\end{array}$ \\
\hline $\begin{array}{l}\text { Chapitre IV } \\
\text { Des phénomènes de la fermenta- } \\
\text { tion, et des moyens de la gouver- } \\
\text { ner (pp. 96-197). }\end{array}$ & $\begin{array}{l}\text { Capítulo V } \\
\text { De la fabricación y conservación } \\
\text { del vino (pp. 118-193) }\end{array}$ & $\begin{array}{l}\text { Es traducción. } \\
\text { La introducción de este } \\
\text { capítulo no se traduce (Chap- } \\
\text { tal pp. 96-99). }\end{array}$ \\
\hline $\begin{array}{l}\text { Section première } \\
\text { Des causes qui influent sur la } \\
\text { fermentation (pp. 100-131) }\end{array}$ & $\begin{array}{l}\text { Artículo Primero } \\
\text { De las causas que influyen en la } \\
\text { fermentación (pp. 118-135). }\end{array}$ & $\begin{array}{l}\text { Es traducción. } \\
\text { Coincidencia plena. }\end{array}$ \\
\hline $\begin{array}{l}\text { Article Première } \\
\text { De l'influence de la température } \\
\text { sur la fermentation (pp. 100-105). }\end{array}$ & $\begin{array}{l}\text { S. I } \\
\text { Del influjo de la temperatura en la } \\
\text { fermentación (pp. 118-121). }\end{array}$ & $\begin{array}{l}\text { Es traducción. } \\
\text { Coincidencia plena. }\end{array}$ \\
\hline $\begin{array}{l}\text { Article II } \\
\text { De l'influence de l'aire dans la } \\
\text { fermentation (106-112). }\end{array}$ & $\begin{array}{l}\text { S. II } \\
\text { Del influjo del aire en la fermenta- } \\
\text { ción (pp. 121-124). }\end{array}$ & $\begin{array}{l}\text { Es traducción. } \\
\text { Coincidencia plena. }\end{array}$ \\
\hline $\begin{array}{l}\text { Article III } \\
\text { De l'influence du volume de la } \\
\text { masse fermentante sur la fermen- } \\
\text { tation (pp. 112-115). }\end{array}$ & $\begin{array}{l}\text { S. III } \\
\text { De lo que influye en la fermenta- } \\
\text { ción la cantidad de la masa fermen- } \\
\text { tante (pp. 125-126). }\end{array}$ & $\begin{array}{l}\text { Es traducción. } \\
\text { Coincidencia plena. }\end{array}$ \\
\hline $\begin{array}{l}\text { Article IV } \\
\text { De l'influence des principes } \\
\text { constituans du moût sur la fer- } \\
\text { mentation (115-131). }\end{array}$ & $\begin{array}{l}\text { S. IV } \\
\text { Del influjo de los principios consti- } \\
\text { tutivos del mosto en la fermenta- } \\
\text { ción (pp. 126-135). }\end{array}$ & $\begin{array}{l}\text { Es traducción. } \\
\text { Coincidencia plena. }\end{array}$ \\
\hline $\begin{array}{l}\text { Section II } \\
\text { Des produits de la fermentation } \\
\text { (pp. 132-151). }\end{array}$ & $\begin{array}{l}\text { Artículo II } \\
\text { De los productos de la fermentación } \\
\text { (pp. 135-148). }\end{array}$ & $\begin{array}{l}\text { Es traducción. } \\
\text { Coincidencia plena. }\end{array}$ \\
\hline $\begin{array}{l}\text { Article Premier } \\
\text { De la production de chaleur (pp. } \\
\text { 135-137). }\end{array}$ & $\begin{array}{l}\text { S. I } \\
\text { De la producción del calórico (pp. } \\
\text { 137-138) }\end{array}$ & $\begin{array}{l}\text { Es traducción. } \\
\text { Coincidencia plena. }\end{array}$ \\
\hline $\begin{array}{l}\text { Article II } \\
\text { Du dégagement de l'acide carbo- } \\
\text { nique (pp. 137-144). }\end{array}$ & $\begin{array}{l}\text { S. II } \\
\text { Del desprendimiento del ácido } \\
\text { carbónico (pp. 138-143). }\end{array}$ & $\begin{array}{l}\text { Es traducción. } \\
\text { Coincidencia plena. }\end{array}$ \\
\hline $\begin{array}{l}\text { Article III } \\
\text { De la formation de l'alcool (145- } \\
\text { 149). }\end{array}$ & $\begin{array}{l}\text { S. III } \\
\text { De la formación del alcohol (143- } \\
\text { 145). }\end{array}$ & $\begin{array}{l}\text { Es traducción. } \\
\text { Coincidencia plena. }\end{array}$ \\
\hline $\begin{array}{l}\text { Article IV } \\
\text { De la coloration de la liqueur } \\
\text { vineuse (149-151). }\end{array}$ & $\begin{array}{l}\text { S. IV } \\
\text { De la coloración del licor vinoso } \\
(145-147) .\end{array}$ & $\begin{array}{l}\text { Es traducción. } \\
\text { Carbonell intercala un frag- } \\
\text { mento (pp. 147-148) que no } \\
\text { está en el original. }\end{array}$ \\
\hline $\begin{array}{l}\text { Section III } \\
\text { Des moyens de gouverner la } \\
\text { fermentation (pp. 148-173). }\end{array}$ & $\begin{array}{l}\text { Artículo III } \\
\text { De los medios de gobernar la fer- } \\
\text { mentación (pp. 148-160). }\end{array}$ & $\begin{array}{l}\text { Es traducción. } \\
\text { Coincidencia plena. }\end{array}$ \\
\hline $\begin{array}{l}\text { Section IV } \\
\text { De la théorie de la fermentation } \\
\text { (pp. 173-185). }\end{array}$ & $\begin{array}{l}\text { Artículo IV } \\
\text { De la teoría de la fermentación (pp. } \\
\text { 160-167). }\end{array}$ & $\begin{array}{l}\text { Es traducción. } \\
\text { Coincidencia plena, salvo una } \\
\text { nota incluida por Carbonell al } \\
\text { final del artículo (p. 167). } \\
\end{array}$ \\
\hline Expériences sur la fermenta- & Experimentos de Mr. Poitevin & Es traducción. \\
\hline
\end{tabular}




\begin{tabular}{|c|c|c|}
\hline $\begin{array}{l}\text { tion vineuse par M. Poitevin } \\
\text { (pp. 186-197). }\end{array}$ & $\begin{array}{l}\text { acerca de la fermentación vinosa } \\
\text { (pp. 168-178). }\end{array}$ & Coincidencia plena. \\
\hline $\begin{array}{l}\text { Chapitre V } \\
\text { Du temps et des moyens de } \\
\text { décuver (pp. 198-218). }\end{array}$ & $\begin{array}{l}\text { Artículo V } \\
\text { Del tiempo y medios de sacar el } \\
\text { vino de los lagares (pp. 179-193). }\end{array}$ & $\begin{array}{l}\text { Es traducción. } \\
\text { Coincidencia casi plena. } \\
\text { Carbonell introduce tres } \\
\text { notas a pie de página (pp. } \\
\text { 182-185/pp. 191-192). } \\
\text { Además Carbonell no tra- } \\
\text { duce un fragmento de Chap- } \\
\text { tal (pp. 210-213), pues los } \\
\text { contenidos ya aparecen en } \\
\text { el capítulo III, haciendo } \\
\text { remisión al mismo. }\end{array}$ \\
\hline $\begin{array}{l}\text { Chapitre VI } \\
\text { De la manière de gouverner les } \\
\text { vins dans les tonneaux (pp. 218- } \\
\text { 248). }\end{array}$ & $\begin{array}{l}\text { Capítulo VI } \\
\text { De las preparaciones diversas para } \\
\text { mejorar el vino y obtenerle de varias } \\
\text { calidades, o del modo de cuidarle en } \\
\text { los toneles (pp. 193-207). }\end{array}$ & $\begin{array}{l}\text { Es traducción. } \\
\text { Coincidencia plena. El título } \\
\text { del capítulo es más extenso } \\
\text { que en el original. }\end{array}$ \\
\hline $\begin{array}{l}\text { Section première } \\
\text { Du soufrage des vins (pp. 226- } \\
\text { 230). }\end{array}$ & $\begin{array}{l}\text { S. I } \\
\text { De la operación de azufrar los vinos } \\
\text { (pp. 197-200). }\end{array}$ & $\begin{array}{l}\text { Es traducción. } \\
\text { Coincidencia plena. Carbo- } \\
\text { nell introduce únicamente } \\
\text { una nota a pie de página } \\
\text { haciendo remisión al cap. III } \\
\text { de su libro (pp. 198-199). }\end{array}$ \\
\hline $\begin{array}{l}\text { Section II } \\
\text { Du soutirage des vins (pp. 230- } \\
\text { 235). }\end{array}$ & $\begin{array}{l}\text { S. II } \\
\text { Del trasiego de los vinos (pp. 200- } \\
\text { 203). }\end{array}$ & $\begin{array}{l}\text { Es traducción. } \\
\text { Coincidencia plena. }\end{array}$ \\
\hline $\begin{array}{l}\text { Section III } \\
\text { Du collage des vins (pp. 235- } \\
\text { 241). }\end{array}$ & $\begin{array}{l}\text { S. III } \\
\text { De la clarificación del vino por } \\
\text { medio de la cola (pp. 203-207). }\end{array}$ & $\begin{array}{l}\text { Es traducción. } \\
\text { Coincidencia plena. }\end{array}$ \\
\hline $\begin{array}{l}\text { Section IV } \\
\text { Des vasseaux propres à conserver } \\
\text { les vins (pp. 241-248). }\end{array}$ & & $\begin{array}{l}\text { No se traduce. } \\
\text { Carbonell no lo traduce, pues } \\
\text { sus contenidos ya están en su } \\
\text { capítulo III. }\end{array}$ \\
\hline $\begin{array}{l}\text { Chapitre VII } \\
\text { Des dégustations et des altéra- } \\
\text { tions spontánées du vin (pp. 248- } \\
\text { 254). }\end{array}$ & $\begin{array}{l}\text { Capítulo VII } \\
\text { De las alteraciones espontáneas del } \\
\text { vino, y del modo de remediarlas } \\
\text { (pp. 208-211). }\end{array}$ & $\begin{array}{l}\text { Es traducción. } \\
\text { Coincidencia plena. }\end{array}$ \\
\hline $\begin{array}{l}\text { Section Première } \\
\text { De la maladie du vin appelée } \\
\text { graisse (pp. 254-259). }\end{array}$ & $\begin{array}{l}\text { S. I } \\
\text { De la enfermedad o alteración que } \\
\text { sufre el vino cuando espesa como } \\
\text { aceite (pp. 211-215). }\end{array}$ & $\begin{array}{l}\text { Es traducción. } \\
\text { Coincidencia plena. }\end{array}$ \\
\hline $\begin{array}{l}\text { Section II } \\
\text { De l'acescense spontanée du vin } \\
\text { (pp. 259-268). }\end{array}$ & $\begin{array}{l}\text { S. II } \\
\text { De la alteración o enfermedad de } \\
\text { agriarse el vino espontáneamente } \\
\text { (pp. 215-221). }\end{array}$ & $\begin{array}{l}\text { Es traducción. } \\
\text { Coincidencia plena }\end{array}$ \\
\hline \multirow[t]{2}{*}{$\begin{array}{l}\text { Section III } \\
\text { Des quelques autres altérations } \\
\text { du vin (pp. 269-272). } \\
\end{array}$} & $\begin{array}{l}\text { S. III } \\
\text { De algunas otras alteraciones es- } \\
\text { pontáneas del vino (pp. 221-224). }\end{array}$ & $\begin{array}{l}\text { Es traducción. } \\
\text { Coincidencia plena }\end{array}$ \\
\hline & $\begin{array}{l}\text { Capítulo VIII } \\
\text { De los vinos artificiales (224-231). }\end{array}$ & Original. \\
\hline
\end{tabular}




\begin{tabular}{|c|c|c|}
\hline & $\begin{array}{l}\text { Capítulo IX } \\
\text { De la policía judicial química del } \\
\text { vino (pp. 231-251) }\end{array}$ & Original. \\
\hline $\begin{array}{l}\text { Chapitre VIII } \\
\text { De l'acétification, ou de la fabri- } \\
\text { cation du vinaigre (pp. 273-310). }\end{array}$ & $\begin{array}{l}\text { Noticia acerca la fabricación del } \\
\text { vinagre (pp. 252-278). }\end{array}$ & $\begin{array}{l}\text { Es traducción. } \\
\text { Coincidencia plena, salvo la } \\
\text { primera parte de la frase con } \\
\text { la que se inicia la Noticia. }\end{array}$ \\
\hline $\begin{array}{l}\text { Section Première } \\
\text { De la fabrication du vinaigre de } \\
\text { vin (pp. 284-289). }\end{array}$ & $\begin{array}{l}\text { S. I } \\
\text { De la fabricación del vinagre hecho } \\
\text { con el vino (pp. 258-261). }\end{array}$ & $\begin{array}{l}\text { Es traducción. } \\
\text { Coincidencia plena. }\end{array}$ \\
\hline $\begin{array}{l}\text { Section II } \\
\text { De la fabrication du vinaigre de } \\
\text { bière (pp. 289-295). }\end{array}$ & $\begin{array}{l}\text { S. II } \\
\text { De la fabricación del vinagre de } \\
\text { cerveza (pp. 262-265). }\end{array}$ & $\begin{array}{l}\text { Es traducción. } \\
\text { Coincidencia plena. }\end{array}$ \\
\hline \multirow[t]{5}{*}{$\begin{array}{l}\text { Section III } \\
\text { De la fabrication du vinaigre par } \\
\text { la distillation des substances } \\
\text { végétales et animales (pp. 296- } \\
\text { 310). }\end{array}$} & $\begin{array}{l}\text { S. III } \\
\text { De la fabricación del vinagre obte- } \\
\text { nido por la destilación de las sus- } \\
\text { tancias vegetales y animales (pp. } \\
\text { 266-274). }\end{array}$ & $\begin{array}{l}\text { Es traducción. } \\
\text { Coincidencia plena. }\end{array}$ \\
\hline & $\begin{array}{l}\text { S. IV } \\
\text { Policía judiciaria química del vina- } \\
\text { gre (pp. 274-278). }\end{array}$ & Original. \\
\hline & $\begin{array}{l}\text { Apéndice al arte de hacer y con- } \\
\text { servar el vino (pp. 279-289). }\end{array}$ & Original. \\
\hline & $\begin{array}{l}\text { S. I } \\
\text { Acerca de la construcción más } \\
\text { ventajosa de las tinas o lagares (pp. } \\
\text { 279-286). }\end{array}$ & Original. \\
\hline & $\begin{array}{l}\text { S. II } \\
\text { Descripción de una máquina para } \\
\text { estrujar las uvas con facilidad y } \\
\text { prontitud, inventada por el Sr. Gerin } \\
\text { maquinista en Montpeller (pp. 286- } \\
\text { 289). }\end{array}$ & Original. \\
\hline $\begin{array}{l}\text { Chapitre IX } \\
\text { Des vertus du vin (pp. 311-318). }\end{array}$ & & No se traduce. \\
\hline \multirow[t]{2}{*}{$\begin{array}{l}\text { Chapitre X } \\
\text { Des principes contenus dans le vin } \\
\text { et de sa distillation (pp. 318-367). }\end{array}$} & & No se traduce. \\
\hline & $\begin{array}{l}\text { Adición al apéndice del Arte de } \\
\text { hacer y conservar el vino (pp. 1- } \\
\text { 16). }\end{array}$ & Original. \\
\hline
\end{tabular}

\section{ANEXO II}

FRAGMENTO 1 (Chaptal, 1796: 47-48; Carbonell, 1820: 54-55)

\section{De l'influence de la culture sur le raisin}

Dans la Florida, en Amérique, et dans presque toutes les parties du Pérou, la vigne croît naturellement. Dans le midi même de la France, les haies sont presque toutes garnies de vignes sauvages : le raisin en est toujours plus petit; et, quoiqu'il parvienne à maturité, il n'a jamais le goût exquis que possède le raisin cultivé. La vigne est donc l'ouvrage de la nature, mais l'art en a per- 
fectionné le produit en en soignant la culture. La différence qui existe aujourd'hui entre la vigne cultivée et la vigne sauvage, est la même que celle que l'art a établie entre les légumes cultivés dans nos jardins et quelques-uns de ces mêmes légumes croissant au hasard dans les champs.

\section{Del influjo del cultivo sobre las uvas}

En la Florida, en la América, y en casi todos los puntos del Perú la viña crece naturalmente. En el mediodía de la Francia los vallados están circundados de vides salvajes; y aunque llegan a madurarse, jamás tienen el gusto exquisito de las uvas cultivadas. La viña es pues obra de la naturaleza; pero el arte ha perfeccionado su producto procurando su cultivo. La diferencia que hay entre la viña cultivada y la viña salvaje, es la misma, que la que el arte establece entre las legumbres cultivadas en nuestros jardines, y algunas de estas mismas que por casualidad se crían en los campos.

\section{FRAGMENTO 2 (Chaptal, 1796: 137; Carbonell, 1820: 138)}

\section{Du dégagement de l'acide carbonique}

Le gaz acide carbonique qui se dégage de la vendange, et ses effets nuisibles à la respiration, sont connus depuis que la fermentation est connue elle-même. Ce gaz s'échappe en bulles de tous les points de la vendange, s'élève dans la masse et vient crever à la surface. Il déplace l'air atmosphérique qui repose sur la vendange; occupe par-tout le vide de la cuve, et deverse ensuite par les bords en se précipitant dans les lieux les plus bas, à raison de sa pésanteur. C'est à la formation de ce gaz qui enlève une portion d'oxigène et de carbone aux príncipes constituans du moût, que nous rapporterons, par la suite, les principaux changements qui surviennent dans la fermentation.

\section{El desprendimiento del ácido carbónico}

El desprendimiento del gas ácido carbónico que se separa de la vendimia y sus efectos dañosos á la respiración, son bien sabidos desde que se conoce la fermentación vinosa. Este gas se desprende en forma de burbujas de todos los puntos de la vendimia, se eleva dentro de la masa fermentante, y va a romper en la superficie de esta. Desaloja al aire atmosférico que se halla sobre la vendimia, ocupa todo el hueco del lagar, y cae después por los bordes de este y se precipita hacia abajo por razón de su peso específico. Á la formación de este gas por medio de la cual se separa una porción de oxígeno y de carbono de los principios constitutivos del mosto, deben atribuirse conforme haremos ver, las principales mutaciones que se observan durante la fermentación.

FRAGMENTO 3 (Chaptal, 1796: 254-255; Carbonell, 1820: 211-212)

\section{De la maladie du vin appelée graisse}

La graisse est une altération que contractent souvent les vins ; ils perdent leur fluidité naturelle, et filent comment de l'huile: on appelle encore cette dégénération, tourner au gras, graisser, filer, etc.

Les vins très généreux dont le goût était très-sucré, ne tournent jamais au gras. Il n'y a que les vins délicats et peu riches en esprit que graissent.

Les vins faibles, qui ont très peu fermenté, sont les plus disposés à cette maladie.

Les vins faibles, faits avec les raisins égrappés, y sont plus sujets.

Le vin tourne au gras dans les bouteilles les mieux fermées. On n'en est que trop convaincu dans la Champagne et la Bourgogne, où toute la récolte contracte quelquefois cette altération.

De la enfermedad o alteración que sufre el vino cuando se espesa como aceite Los vinos experimentan muchas veces esta alteración: ellos pierden su fluidez natural y se espesan como el aceite: llaman a esta alteración, espesarse el vino, hacer hebra, etc.

Los vinos muy generosos, procedentes de un mosto muy azucarado, jamás se espesan: únicamente los vinos flacos y poco espirituosos son los que están sujetos a padecer esta alteración. Los vinos flacos que han fermentado poco, están muy dispuestos a sufrirla. Los vinos flacos fabricados con las uvas desgranadas o separados del hollejo, están aún más sujetos a la misma.

El vino sufre esta alteración de espesarse en las botellas bien cerradas. Esto es lo que se observa y sucede en Champaña y en Borgoña, en donde algunas veces toda la cosecha sufre este género de alteración. 